\title{
Spatiotemporal multifractal characteristics of electromagnetic radiation in response to deep coal rock bursts
}

\author{
S. Hu ${ }^{1,2}$, E. Wang ${ }^{1,2}$, and X. Liu ${ }^{1}$ \\ ${ }^{1}$ School of Safety Engineering, China University of Mining \& Technology, Xuzhou, China \\ ${ }^{2}$ Key Laboratory of Gas and Fire Control for Coal Mines, Xuzhou, China \\ Correspondence to: E. Wang (weytopcumt@163.com)
}

Received: 3 January 2014 - Published in Nat. Hazards Earth Syst. Sci. Discuss.: 4 April 2014

Revised: 26 June 2014 - Accepted: 8 July 2014 - Published: 15 August 2014

\begin{abstract}
Dynamic collapses of deeply mined coal rocks are severe threats to miners; in order to predict collapses more accurately using electromagnetic radiation (EMR), we investigate the spatiotemporal multifractal characteristics and formation mechanism of EMR induced by underground coal mining. Coal rock in the burst-prone zone often exchanges materials (gas, water and coal) and energy with its environment and gradually transitions from its original stable equilibrium structure to a nonequilibrium dissipative structure with implicit spatiotemporal complexity or multifractal structures, resulting in temporal variation in multifractal EMR. The inherent law of EMR time series during damage evolution was analyzed by using time-varying multifractal theory. Results show that the time-varying multifractal characteristics of EMR are determined by damage evolution processes. Moreover, the dissipated energy caused by the damage evolutions, such as crack propagation, fractal sliding and shearing, can be regarded as the fingerprint of various EMR micro-mechanics. The dynamic spatiotemporal multifractal spectrum of EMR considers both spatial (multiple fractures) and temporal (dynamic evolution) characteristics of coal rocks and records the dynamic evolution processes of rock bursts. Thus, it can be used to evaluate the coal deformation and fracture process. The study is of significance for us to understand the EMR mechanism in detail and to increase the accuracy of the EMR method in forecasting dynamic disasters.
\end{abstract}

\section{Introduction}

With coal mining deepening, dynamic disasters such as rock burst and roof collapse become increasingly severe, threatening mine safety and efficient production. Monitoring and forecasting coal rock dynamic disasters have become the key issues to be resolved. Since the discovery of electromagnetic emission (EM) from materials during their deformation and fracture (Nitsan, 1977; Warwick et al., 1982; Ogawa et al., 1985; Brady and Rowell, 1986; Cress et al., 1987), electromagnetic radiation (EMR) has been widely applied to monitor and forecast seismic and dynamic disasters (rock burst, wall caving, coal and gas outburst) (Warwick et al., 1982; Vallianatos and Nomikos, 1998; Vallianatos and Tzanis, 1998, 2003; Tzanis and Vallianatos, 2001, 2002; Vallianatos et al., 2004; Eftaxias et al., 2003; Frid and Vozoff, 2005; Contoyiannis et al., 2005; Wang et al., 2012; He et al., 2012; Potirakis et al., 2012). The critical phenomena and fractal characteristics of EMR have been observed before seismic or rock fracture (Hayakawa et al., 2004; Rabinovitch et al., 2001; Kapiris et al., 2003; Uritsky et al., 2004; Kapiris et al., 2004a, b; Gotoh et al., 2004; Eftaxias et al., 2007; Muto et al., 2007). Some scholars have considered that the critical phenomena of EM emission are related to crack opening and expansion, as well as fractal structure (Hayakawa et al., 2004; Nanjo and Nagahama, 2004a, b; Kapiris et al., 2004a, b; Morgounov and Malzev, 2007; Kawada et al., 2007; Minadakis et al., 2012). Uritsky et al. (2004) proposed a simple framework for modeling ultra-low frequency (ULF) electromagnetic emission signals associated with abrupt changes in the large-scale geometry of stress distribution before characteristic seismic events. Kapiris et 
al. (2004b) found that the critical characteristics of EM were determined by the nonlinear multi-fracture of discrete materials. From the thermodynamic view of irreversible damage evolution, Kawada et al. (2007) introduced dipoles and explained the critical phenomena of EM emission and their timescale invariance theoretically. This research is of significance for the explanation of EMR critical characteristics before seismic events or during rock fracture.

Moreover, EMR was emitted before seismic events or rock failure process. EMR has critical characteristics, and experimental and in situ observation data also show that the EMR time series exhibits characteristics of the time-varying fractal dimension, change in entropy and multifractals (Wei et al., 2005; Eftaxias et al., 2007; Potirakis et al., 2012; Minadakis et al., 2012). Eftaxias et al. (2007) analyzed the fractal spectra of a large number of pre-burst EMR signals and nonlinear approximate entropy and found that the fractal dimension and approximate entropy changed with time. In addition, many scholars have performed multidisciplinary analyses of criticality (Eftaxias et al., 2003; Kapiris et al., 2004a; Contoyiannis et al., 2005) and complexity (Eftaxias et al., 2007), and found that during coal rock fracturing, EMR intensity increased in accordance with a power law, while before global ruptures, EMR underwent a very complex, intensity-increasing process. Researchers such as Vallianatos, etc. (Vallianatos and Nomikos, 1998; Vallianatos and Tzanis, 1998, 2003; Tzanis and Vallianatos, 2001, 2002; Vallianatos et al., 2004) proved a promising effect that is ubiquitous during brittle rock failure: the motion of charged edge dislocations (MCD) during crack opening and propagation (microfracturing). Vallianatos et al. (2004) assumed that when ionic crystals and rocks undergo such drastic changes, more than one electrification mechanism may be operative. These findings imply that EM emission is caused jointly by many mechanisms and changes dynamically with the system developing. The solid deformation and fracture represented a multilevel self-organized process, in which changes in internal structure at micro-, meso- and macro-levels are organically linked. During the damage evolution of such a system, cracks on various scales coexist and occur simultaneously, resulting in the emission of very complex EMR. In short, linear and multiple fractures of solid materials will lead to a dynamic, nonlinear EMR fluctuation.

Coal rocks, as a natural, discrete medium, and sedimentary materials have many defects such as stratification, joints, pores, fractures, etc. Dynamic disasters, such as rock burst and roof collapse, are instantaneous, instable phenomena occurring in coal rocks under the effects of external physical and chemical factors or stress. Along with the instable process, EMR is generated and emitted. Studies have found that EMR intensity and activity are significantly enhanced with rock burst risks increasing (Frid and Vozoff, 2005; Wang et al., 2012; He et al., 2012). Field observations and experimental studies also showed that rock burst is an open and dissipative system with complex structures and behavior and that it can exchange energy with the external environment (Lu et al., 2004, 2007). Song et al. (2012) have applied the dissipativestructure theory to study energy accumulation, dissipation and entropy changes during coal and rock deformation and failure and found that energy accumulation and dissipation possess self-organized criticality (SOC). Similar to the multiple nonlinear rupture process of discrete media, EMR generation is accompanied by coal rock dynamic evolution, and carries the fingerprint of system instability or rock burst; thus, it occurs before coal rock failure and burst. However, investigating the electromagnetic responses to underground geodynamic processes, particularly the pre-seismic and seismic processes, is a challenging task in modern geophysics. Increasing evidence has shown that it is difficult to quantitatively describe seismic electromagnetic (SEM) phenomena by "linear" models using "averaged" parameters. Because a coal rock system is highly inhomogeneous (on all scales) and anisotropic, the effective way to describe it is via nonlinear theory.

Experimental and theoretical evidence has shown that disordered media in a critical, instable phase possesses an implicit spatiotemporal complexity which could form fractal structures on multiple scales, which is similar to the nonequilibrium phase transition process (Eftaxias et al., 2003, 2007). Thus, the fractal dimension and its relevant index can be used as a indicator to analyze the characteristics of the system. For example, spatiotemporal dynamical characteristics of the instable, nonlinear system during the dynamic evolution process could be revealed using a windowed multifractal singular spectrum and a temporal multifractal spectrum (Wang and Zhu, 2001; Masao and Takehisa, 2007; Gang et al., 2012).

Real-time online EMR monitoring is the basis for studying the mechanisms and characteristics of EMR from coal rock bursts during underground coal mining. We have previously developed a KBD7 (Kuang yong Ben an Dian ci fu she) online real-time EMR monitoring instrument and widely applied it to predict coal and gas outbursts and rock bursts in mines and mining faces (Wang, 1997; Wang et al., 2011, 2012; He et al., 2012). Based on real-time field monitoring data, in this paper, we analyze an EMR multifractal spectrum to explore the mechanisms for EMR generation in the process of rock burst evolution, investigate the temporal response characteristics of EMR generated by coal rock burst during underground mining, reveal the nature of nonlinear, dynamical EMR changes, and further discuss the EMR multifractal practicability. The results are of great significance for further understanding the rock burst mechanisms and improving the reliability of EMR monitoring and early warning. 


\section{Instrumentation}

\subsection{EMR data acquisition system}

EM from coal rock is the electromagnetic energy generated during the deformation and failure of coal rock and is closely related to the process of coal rock deformation and failure. EMR could comprehensively reflect the effects of the main factors on dynamic disasters, such as rock burst and roof collapse among others, and on the degree of coal rock deformation and fracture under load. To this end, we designed and developed an EMR monitoring instrument and used it to measure the EMR generated due to coal rock deformation and failure in the working face and to predict coal rock dynamic disasters.

Figure 1 displays the KBD7 online EMR monitoring instrument; the detailed specifications refer to related literature (Wang, 1997; Wang et al., 2011, 2012; He et al., 2012). It consists of a highly sensitive, directional reception antenna, a host computer, a remote communication interface and a transmission cable and has major functions including data collection, conversion, storage and processing, as well as communication and giving a warning. In addition, the explosion-proof design guarantees its security in applications. Due to the wide band of electromagnetic radiation in underground mining space, we chose a high-sensitivity wideband directional magnet receiver antenna with the following characteristics: frequency bandwidth: wideband (1$500 \mathrm{kHz}$ ); test mode: noncontact orientation test; antenna size: diameter $70 \times 300 \mathrm{~mm}$. The monitoring signal is amplified by the additional preamplifier. Figure 2 shows the KBD7 coal and rock dynamic disasters noncontact EMR monitor principle diagram.

The signal collection, conversion, processing, storage and output are done automatically and continuously by the KBD7 monitoring system. The EMR signals received by the antenna can not be processed directly because they are too weak. They are amplified by the preamplifier, converted by A/D converter and deposited in the cache. The data in the cache could be read out by CPU and stored in data memory. The monitoring data can be displayed on the LCD display in real time. The monitor can work either alone through an external power supply or together with the KJ (Kuang Jing) coal mine safety monitoring system. A customized application software is installed on the computer at the monitoring center and control terminals and can be easily used for real-time EMR acquisition, display and analysis.

\subsection{Equipment installation method}

To use the instrument, one needs to first set the EMRreceiving antenna to face key monitoring areas on the working face, such as the stress-concentrated area, advancing face and geologic structure zone. The EMR signal attenuation can be influenced by electrical parameters of coal and rock

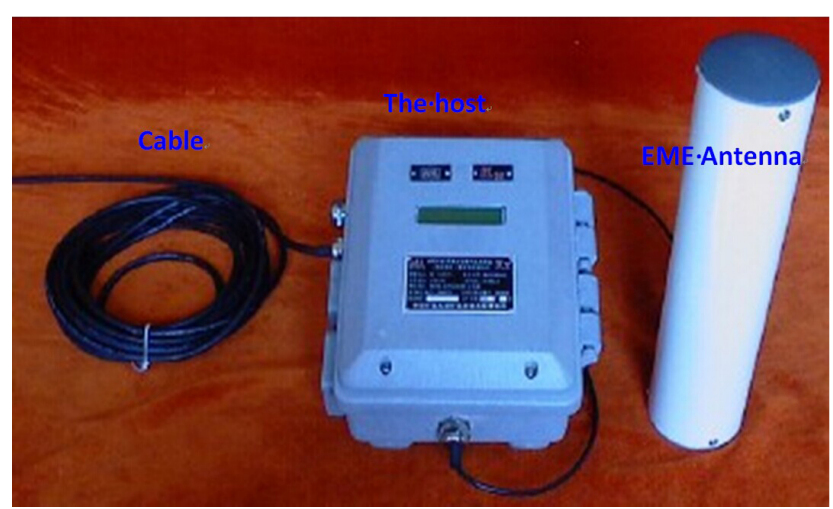

Figure 1. KBD7 EMR monitoring instrument.

(resistivity/conductivity), coal mechanical parameters, composition, the stress state of the coal seam, and gas and moisture content. Therefore, the signals attenuate sharply when the signals propagate outward through the surrounding coal and rock. The monitoring areas of EMR are limited due to the high signal attenuation. EMR signals generated by coal and rock deformation and fracture mainly consist of lowfrequency signals. According to electromagnetic theory, the frequency of the maximum power point of EMR changes over the distance from the radiation source, and the frequency varies inversely as the square of the distance. The frequency of the maximum power radiation is determined by spectrum analysis, allowing us to predict the distance from the EMR radiation source effectively. The specific derivation was provided in literature (Wang Enyuan et al., 2009). All of the above factors would affect the EMR antenna layout.

According to the literature (Wang et al., 2009), the most suitable distance between the antenna and the measured area is equal to or less than $5 \mathrm{~m}$, depending upon the size of the monitored area, which has to be located in the monitoring scope of the antenna. The monitoring distance or range of each antenna is about $20 \mathrm{~m}$; therefore the measuring points are set every 10 to $20 \mathrm{~m}$ in the working face. For the zones of greater rock burst risk, the measuring points should be arranged as closely as possible. The monitor can work either alone through an external power supply or together with the KJ coal mine safety monitoring system (see Fig. 3).

In this study, the sampling frequency of our antenna is $25 \mathrm{kHz} \mathrm{s}^{-1}$, which is close to the frequency of the coal rock EMR. The system is set up to record and store EMR data every $15 \mathrm{~s}$ and the whole signal transmission process predicting rock burst and roof collapse is as follows: (1) receive EMR signals by directional antenna; (2) after amplification, store the electric signals in the buffer zone, filter noises and perform analog-digital transformation; (3) conduct statistical analyses and output the standard signals (1-5 mA, 4-20 mA or $200-1000 \mathrm{~Hz}$ ); and (4) transfer data to the central computer via the KJ31 (KJ coal mine safety monitoring system) coal mine safety monitoring system. 


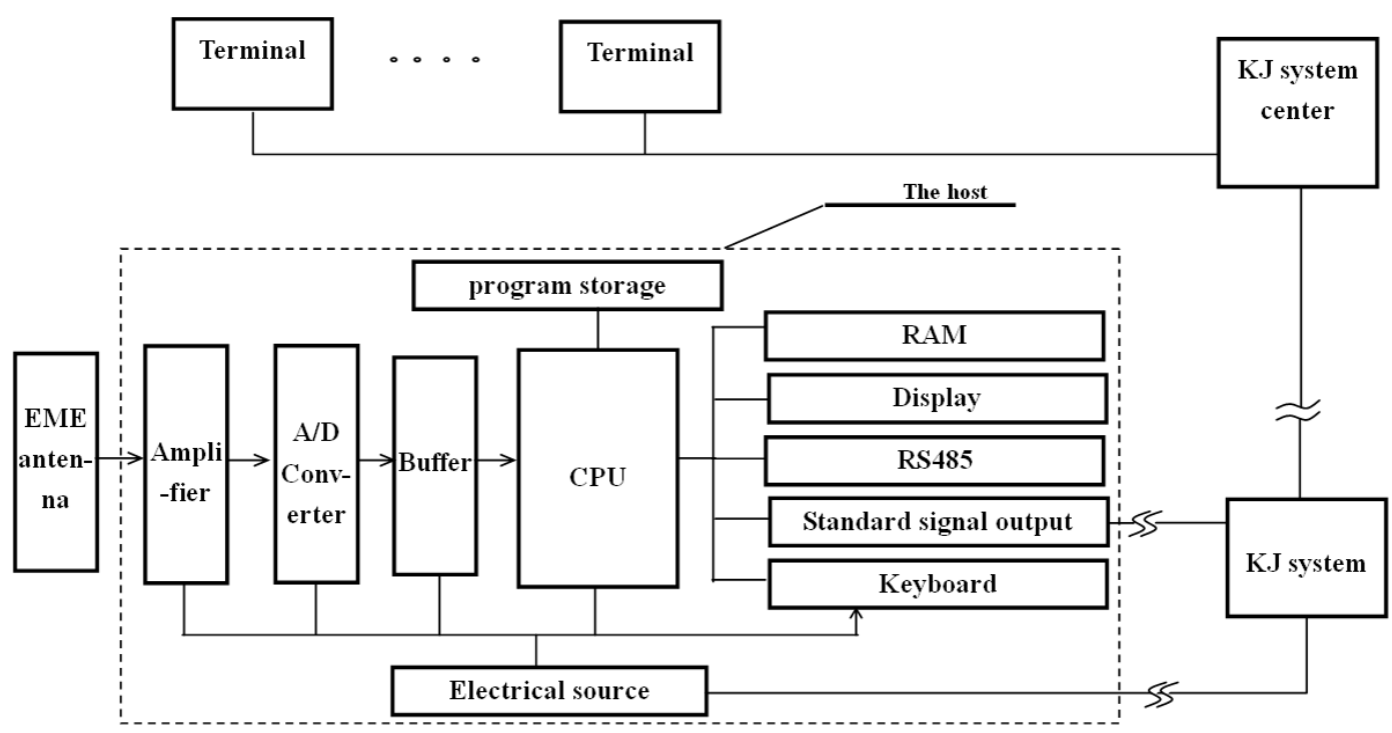

Figure 2. KBD7 Coal and rock dynamic disaster noncontact EMR (Electromagnetic radiation Emission) monitor principle diagram.

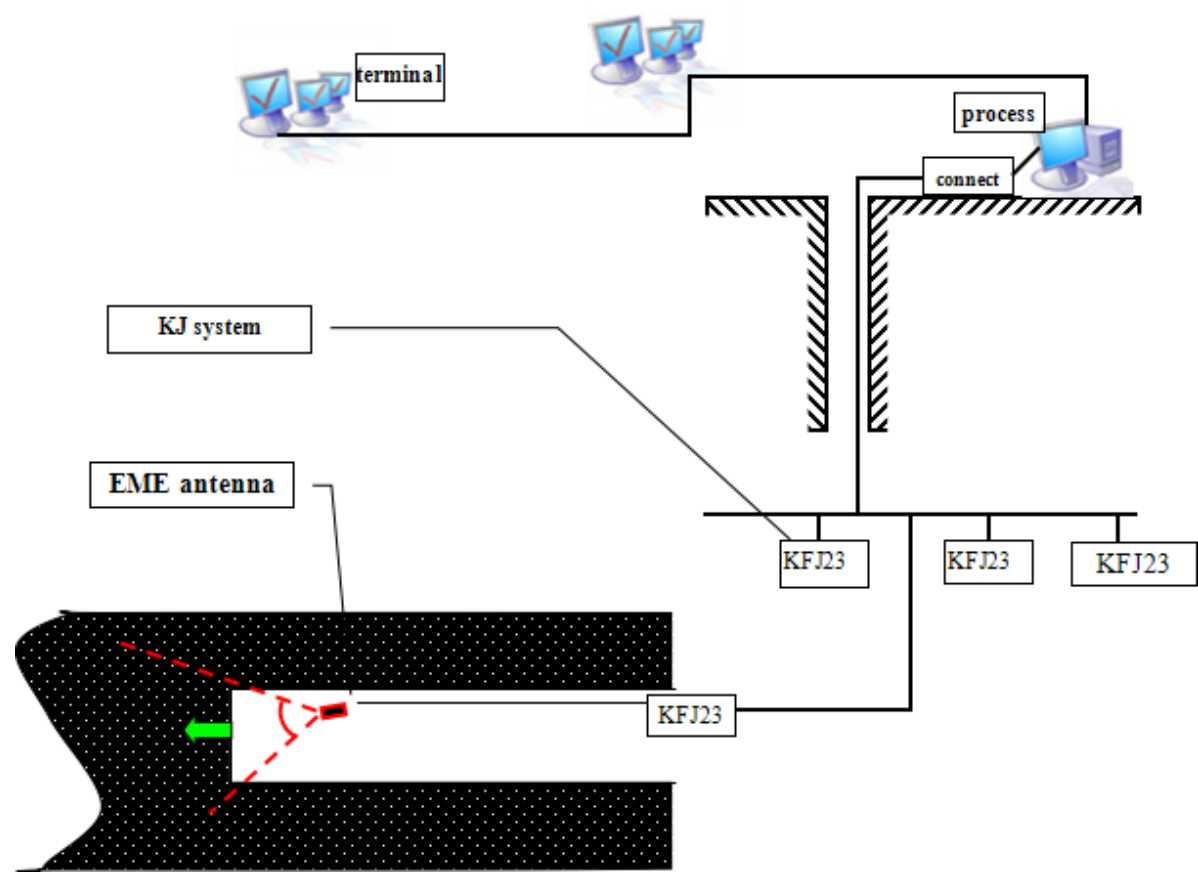

Figure 3. Instrumentation arrangement of the KBD7 EMR (Electromagnetic radiation Emission) monitor system.

\section{Observation results}

The first field investigation on rock burst was conducted at Coal Mine A located in the city of Yi Ma, Henan Province, China. The coal mine was founded in 1958; now the mine production capacity is 1.48 million tons per year. The rock burst occurred during excavation at mining face no. 23150 of the bolted roadway before dawn on 12 March 2013, causing great damage to the roadway over a space of $50 \mathrm{~m}$, slight roof sinking, $0.8 \mathrm{~m}$ upheaval of the floor, $0.8 \mathrm{~m}$ displacement of both sides of the roadway and deformation of the supports at different degrees. Ground elevation is $+532-+560.2 \mathrm{~m}$, average elevation of the roadway is about $-330 \mathrm{~m}$ and the depth is about $880 \mathrm{~m}$. The coal seam roof is mudstone, $20 \mathrm{~m}$ thick or so, and the bottom is fine-grained sandstone which is 0 $25 \mathrm{~m}$ thick. The compressive strength of the coal is as high as $40 \mathrm{MPa}$. The average thickness of coal seam is about $6.6 \mathrm{~m}$, being strongly prone to bumps. Figure 4 shows the position of the EMR monitoring antenna in the advancing roadway. 


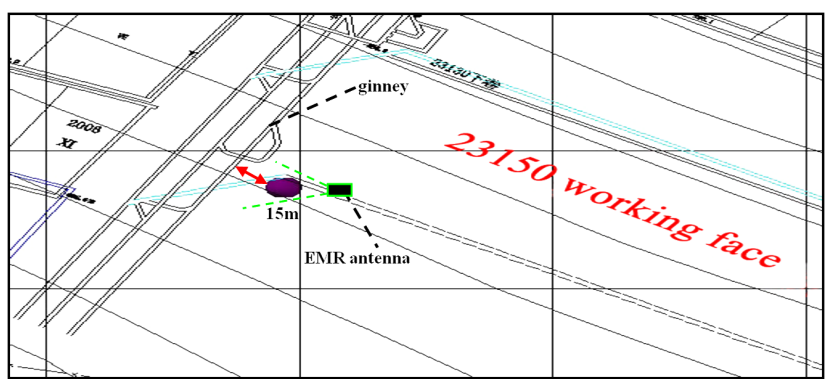

Figure 4. Schematic of EMR monitoring in the mining roadway.

Figure 5 shows the variation of EMR during the excavation of roadway no. 23150. In the rail crisscross location, it is easy to cause the stress concentration. From 6 to 10 March, EMR intensity increased about threefold from its basal level of $18 \mathrm{mV}$ to the peak of $60 \mathrm{mV}$, and the measured EMR varied strongly. (The amplitude of the spikes we refer to here is, in fact, the maximum basic value (the background EMR signals). This kind of processing method can avoid interference signals, but also it is easy to lose true and useful signals. Therefore, in this paper, we study the time-varying multifractal of electromagnetic radiation. This method can analyze the partial, abnormal signals and the overall trends.) The signal intensity decreased to $20 \mathrm{mV}$ on 11 March, and the rock burst occurred in the excavation roadway before dawn on 12 March. Before the rock burst, the signals increased once again and exhibited the supercritical phenomenon from 10 to 11 March, indicating that the mining face had entered the dangerous zone where coal rock was prone to bursts. The rock burst occurred in the advancing roadway 2 days after the appearance of the supercritical phenomenon. In addition, EMR intensity showed a decreased trend two days before the occurrence of the rock burst after reaching its peak.

EMR intensity is affected by different stope backgrounds. The underground space electromagnetic radiation signals, when undisturbed, have their own independent background signal, and the signal is very stable. The production of coal and rock EMR signal is mainly affected by the development and slipping of internal cracks of coal or rock. EME (Electromagnetic radiation Emission) from loaded coal is determined by its mechanical properties, stress level and loading rate (Hu et al., 2013). In general, the higher the coal intensity is, or the greater the stress level or the loading rate, the greater the EMR signals. Therefore, even in the same coal mine, the background EMR signal is different at different working faces. Figure 6 shows the dynamic variation of EMR generated from the coal rock of the bolted roadway at the driving face of Coal Mine B. Coal mine B is located in Hei Long Jiang Province, China. It is currently engaged in deep mining, and the strata condition is serious. Several rock burst accidents have happened. The coal seam is about 8.21-12.94 m thick, and the immediate roof is fine sandstone for 4 to $7 \mathrm{~m}$; directly below, there is siltstone for 4 to $7 \mathrm{~m}$.
Here, EMR intensity was mainly affected by mining-induced stress. The critical value of EMR was $150 \mathrm{mV}$, which was very different from that obtained from the mining roadway of Coal Mine A. (Before EMR monitoring and early warning in an area, generally, we need to monitor the EMR signals of the area for a period of time and determine the background EMR signal strength. According to the underground pressure situation, we determine the critical value of electromagnetic radiation. We use our own algorithms and software, but it is not practical to provide detailed information here; detailed information can be obtained from the following references: Wang, 1997; Wang et al., 2011, 2012; He et al., 2012.) It can be seen from Fig. 6 that the EMR increased noticeably after 08:00 UTC. On 3 November 2011, its basal level exceeded the critical value. At 19:45 UTC on that day, the rock burst occurred in the bolted roadway when EMR was increasing, causing severe deformation of the roadway.

It can be seen from the above two examples that the electromagnetic radiation will be abnormal before rock burst. Electromagnetic radiation of coal or rock is mainly generated by the development of internal cracks of coal or rock and the slip; the higher the coal and rock stress, the greater the intensity of electromagnetic radiation. Literature (Hu et al., 2013) shows that electromagnetic radiation is affected by the mechanical properties of coal, stress level and loading rate. The background electromagnetic radiation signal has obvious differences when the mining face, the mechanical properties of coal, coal face stress state and geological structure are different. In the process of rock burst evolution, the electromagnetic radiation reference signal will be increased; at the same time, the electromagnetic radiation will change intensely. Wang et al. (2011) have proved that rock burst could occur when EMR intensity changes, either increasing or decreasing, making it very difficult to monitor and give an early warning of rock burst. For example, the rock burst in mine A occurred in the process of the electromagnetic radiation reducing, but for mine $\mathrm{B}$, the rock burst occurred in the process of electromagnetic radiation increasing. The process of the rise or fall of electromagnetic radiation conveys much potential information, such as, at a certain moment, the strong change in electromagnetic radiation (on 6 and 7 March, Fig. 5). We can not ignore the "abnormal" signal (it can easily be regarded as an interfering signal). Therefore, it is necessary to further analyze the nature and inherent laws of the nonlinear dynamic characteristics of EMR during the spatiotemporal evolution of rock burst.

By contrast, an EMR time series was also recorded from regions in which no damage developed (see Fig. 7). It can be seen in Fig. 7 that the electromagnetic radiation energy stays at a low level, with a slight fluctuation, in non-rock-bursthazard areas. 


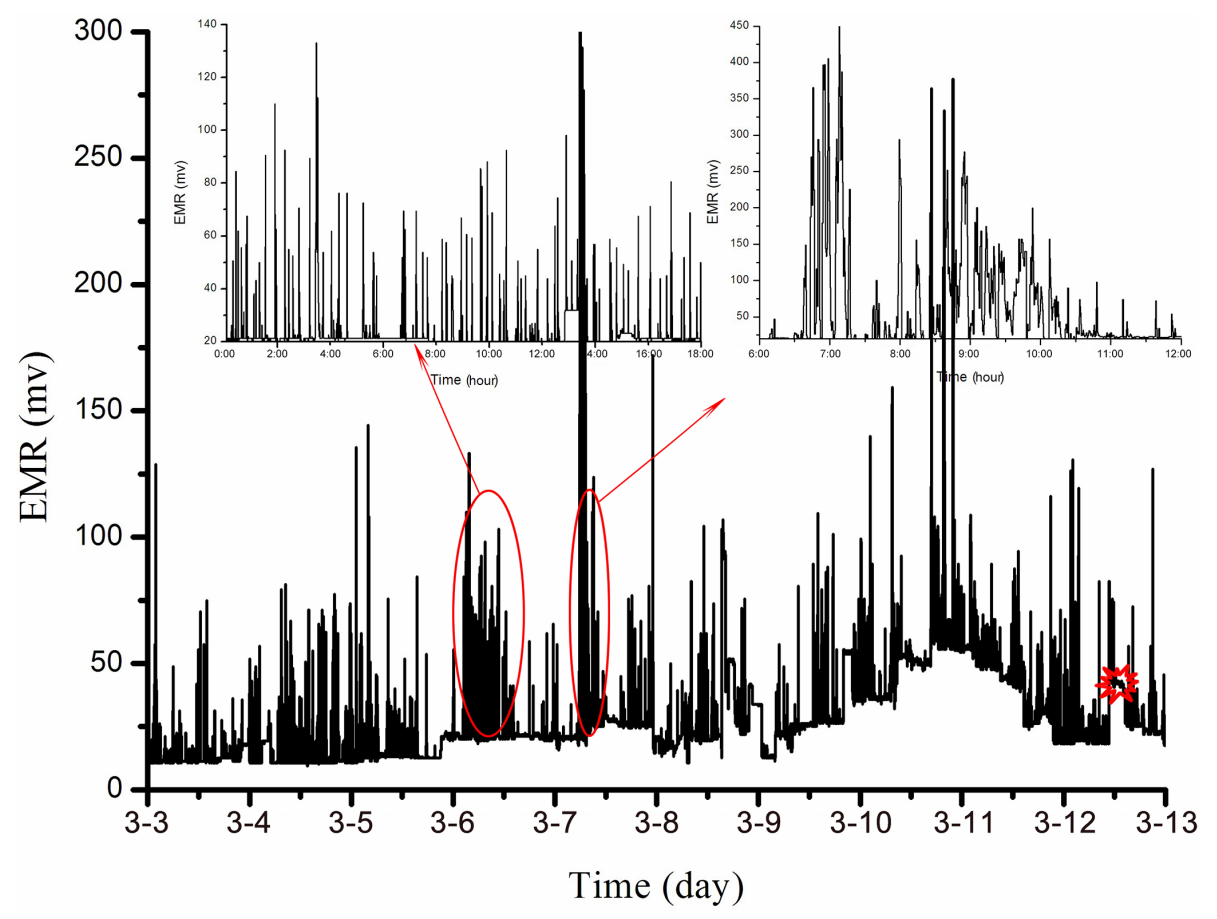

Figure 5. Dynamic variation of EMR from coal rock at the mining roadway of Coal Mine A before and after the rock burst occurred before dawn on 12 March 2013.

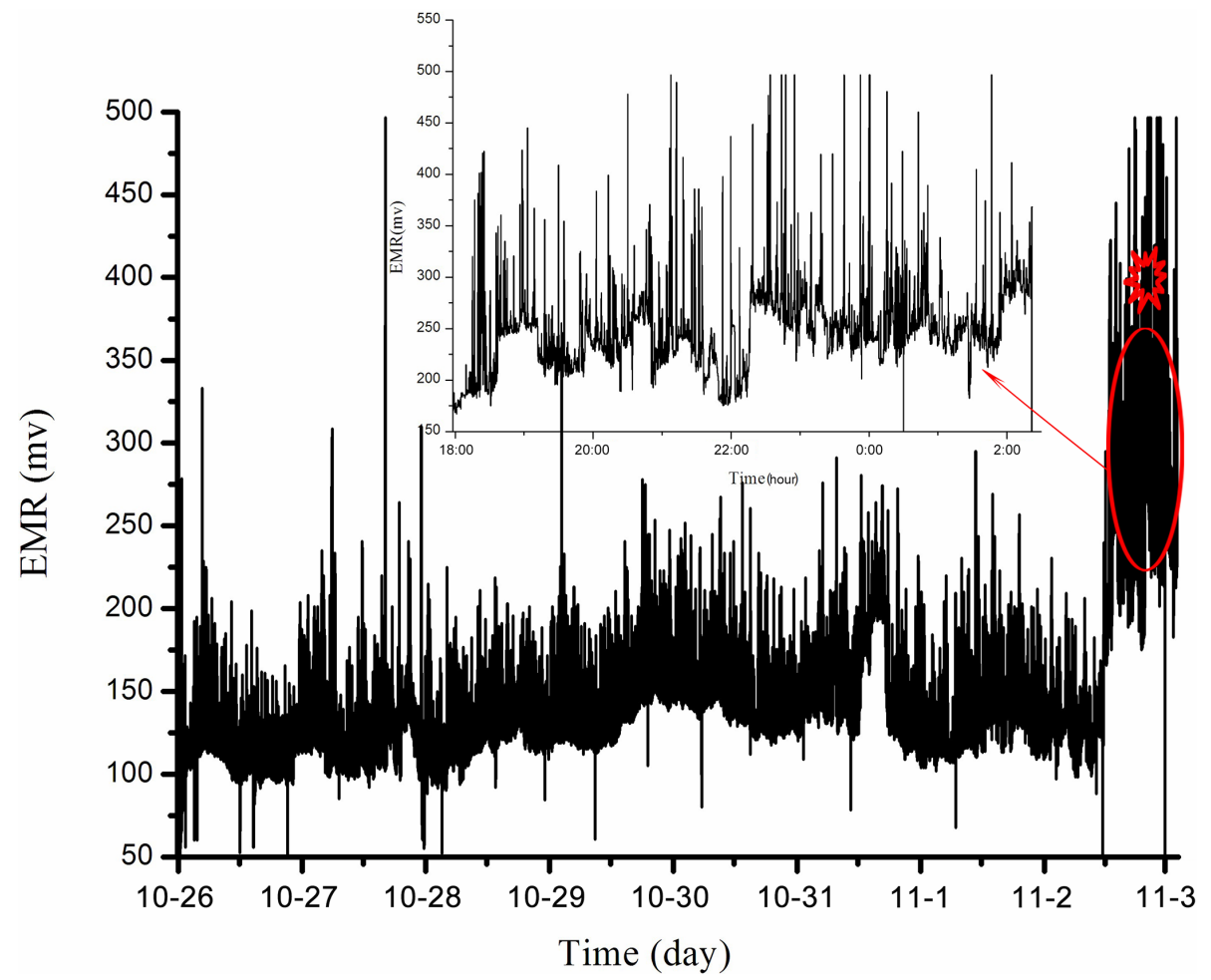

Figure 6. Dynamic variation of EMR generated from the coal rock of the bolted coal roadway in October 2011. 


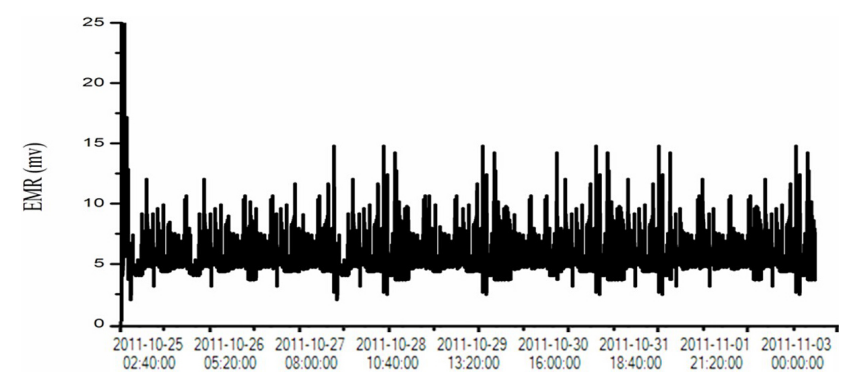

Figure 7. Dynamic variation of EMR generated from regions in which no damage developed.

\section{Temporal characteristics of EMR from coal rock burst}

\subsection{Temporal multifractal spectrum of EMR and its physical meanings}

EMR can reflect the deformation and failure process of coal rocks and reveal spatiotemporal dynamic characteristics of coal- and rock-induced damage. Experimental and theoretical evidence shows that at their critical, instable phase, the spatiotemporal complexity of disordered media will present itself, generally forming a multi-scale fractal structure similar to the transition process of the nonequilibrium phase (Eftaxias et al., 2003, 2007).

Assuming that an EMR time series $\left\{x_{i}\right\}$ can be divided into $N$ subsets of length $\varepsilon$, the probability distribution of each subset is calculated as $\left\{P_{i}(\varepsilon)\right\}$. If the time series has multifractal characteristics, the probability distribution function $\left\{P_{i}(\varepsilon)\right\}$ and divided scale $\varepsilon$ as $\varepsilon \rightarrow 0$ obey the following equation:

$\left\{p_{i}(\varepsilon)\right\} \propto \varepsilon^{\alpha}$,

where $i$ is a sign of the electromagnetic radiation data changing over time, which reflects the amount of electromagnetic radiation data, and $\alpha$ is a constant known as the singularity exponent, controlling the singularity of the probability function $\left\{P_{i}(\varepsilon)\right\}$ and reflecting the various divided scales $\varepsilon$ of the time series and the properties of variables whose probability distribution function changes with the divided scale $\varepsilon$ changing. It reflects the nonuniformity of a subset probability.

If the number of units with the same probability in the subsets marked by $\alpha$ is denoted as $N_{\alpha}(\varepsilon)$, generally speaking, the smaller the divided scale $\varepsilon$ is, the greater the number of subsets obtained. Hence, $N_{\alpha}(\varepsilon)$ increases with $\varepsilon$ decreasing and exhibits the following relationship:

$N_{\alpha}(\varepsilon) \propto \varepsilon^{-f(\alpha)}$,

where $f(\alpha)$ is the frequency of the subset represented by $\alpha$ in the whole subset collection, that is, the fractal dimension of the $\alpha$ subset.
However, practically, gramma is difficult to calculate using the definition. At present, a statistical physics method is generally applied to compute multifractal spectra. First, a partition function, i.e., the statistical moment is defined as follows:

$X_{q}(\varepsilon) \equiv \sum p_{i}(\varepsilon)^{q} \sim \varepsilon^{\tau(q)}$,

where $\tau(q)$ is the quality index, with $-\infty<q<+\infty$. In actual calculation, when $|q|$ reaches a definite value, the multifractal spectrum tends to be stable; after that, even at greater values, generally, the value $q$ is defined within a certain range.

As the equality in Eq. (3) holds true - that is, the defined partition function $X_{q}$ is the power function of the divided scale $\varepsilon$ - the quality index $\tau(q)$ can be obtained by calculating the slope of the double logarithmic curve, $\ln X_{q}(\varepsilon)-\ln \varepsilon$, i.e.,

$\tau(q)=\lim _{\varepsilon \rightarrow 0} \frac{\ln X_{q}(\varepsilon)}{\ln \varepsilon}$.

The ideal, regular multifractal curve, $\ln X_{q}(\varepsilon)-\ln \varepsilon$, satisfies the strict linear relationship, and actual series with multifractal characteristics also exhibit a good linear relationship; otherwise, abnormality will occur as the $q$ value changes.

After performing the Legendre transform of $\tau(q)-q$, we obtained

$\alpha=\frac{\mathrm{d}(\tau(q))}{\mathrm{d} q}=\frac{\mathrm{d}}{\mathrm{d} q}\left(\lim _{\varepsilon \rightarrow 0} \frac{\ln X_{q}(\varepsilon)}{\ln \varepsilon}\right)$

and

$f(\alpha)=\alpha q-\tau(q)$.

The curve $\alpha-f(\alpha)$ composed of $\alpha$ and $f(\alpha)$ is the multifractal spectrum of the calculated series and reflects the unevenly distributed property within time series $\left\{x_{i}\right\}$. $\alpha$ denotes different signal subsets, among which one, represented by $\alpha_{\min }$, corresponds to large signals, while the one represented by $\alpha_{\max }$ corresponds to small signals. Hence, the width of the multifractal spectrum, $\Delta \alpha=\alpha_{\max }-\alpha_{\min }$, can reflect the difference between both signals, $\alpha_{\max }$ and $\alpha_{\min }$, and greater $\Delta \alpha$ means greater difference between both signals. The size of $f(\alpha)$ represents the frequency at which the signal subset of singularity $\alpha$ occurs in the entire loading process. Let $\Delta f=f\left(\alpha_{\max }\right)-f\left(\alpha_{\min }\right)$, then $\Delta f$ reflects the relationship between small and large signal frequencies.

Although multifractal singular spectrum analysis reveals its singularity distribution, it does not take time into account. Thus, it is difficult to describe the dynamic evolution process of instable and nonlinear systems. To reveal spatiotemporal dynamic characteristics of multifractal systems and instable random fractal signals, temporal multifractal spectra or windowed multifractal singular spectra - are put forward (Wang and Zhu, 2001; Masao and Takehisa, 2007; Gang et al., 2012). 
Let the EMR time series be $\left\{y_{i}\right\}$, and its total time length is $T$. In time window $l_{t}$, if the time interval for data acquisition is $\Delta t$, then the EMR time series $X_{m}$ in the time window $l_{t}$ is

$X_{m}=\left\{x_{i}=y_{i} \mid y_{i=a m \Delta t}\left(l_{t}+a m \Delta t\right)\right\}, m=0, \ldots, \frac{T-l_{t}}{a \Delta t}$,

where $a$ is a positive integer reflecting the data renewal rate of the time series in the time window $l_{t} . X_{m}$ is put into Eqs. (1)-(7) to find the $f_{m}(\alpha)-\alpha$ multifractal singular spectrum set and its relevant parameters, while the dynamic fractal singularity index at the moment $T_{m}=l_{t}+\alpha m \Delta \alpha$ is

$\alpha_{m}=\frac{\mathrm{d}\left(\tau_{m}(q)\right)}{\mathrm{d} q}=\frac{d}{d q}\left(\lim _{\varepsilon \rightarrow 0} \frac{\ln X_{q}(\varepsilon)}{\ln \varepsilon}\right)$,

$f_{m}(\alpha)=\alpha_{m} q-\tau_{m}(q)$.

Dynamic changes in multifractal parameters can be used to reveal the differences in microscopic EM emission mechanisms at different stages of coal deformation and failure. $\Delta \alpha_{m}$ indicates the distributive nonuniformity of the studied object and can be used to reflect differences in microscopic EM emission mechanisms of loaded coal. Greater $\Delta \alpha_{m}$ indicates a greater difference among microscopic EM emission mechanisms. $\Delta f_{m}$ is a measure of the ratio difference between the number of great and small events of the studied object and can be used to measure the proportion of strong and weak EM emission mechanisms. $\Delta f_{m}>0$ means that weak EM emission mechanisms are dominant over the strong ones, while $\Delta f_{m}<0$ indicates the strong ones are dominant.

For the spatial variation of the electromagnetic radiation during underground mining, $\Delta \alpha_{m}$ and $\Delta f_{m}$ have clear physical meanings. The EMR released from coal or rock is a result of the combined action of different microradiation mechanisms (Gokhberg et al., 1982; Nagahama and Teisseyre, 1998; Freund et al., 2004; Triantis et al., 2006; Miura and Nakayama, 2001; Muto et al., 2006; Akito and Nobumasa, 2008). Generation of EM signals is related to the dislocation and sliding of coal joints, cracks and lattices, as well as crack development and could lead to dynamic nonlinear changes of EMR. The greater the $\Delta \alpha_{m}$ is, the more obvious the multifractal characteristics of EMR, which suggests an increased difference of EM mechanisms and implies that the coal and rock system is moving from a stable state into a nonlinear acceleration deformation stage, involving reduced stability and increased outburst risk. $\Delta f_{m}$ reflects the difference in proportion of micromechanisms of EMR. EMR is associated with coal or rock dissipation energy (Yao et al., 2010; Song et al., 2012). The greater the dissipated energy rate is (the greater the damage rate is), the greater the EMR intensity. Thus, compared to the strong plastic flow (shear failure), brittle fracture (crack propagation) is a strong microcosmic mechanism of radiation. $\Delta f_{m}>0$ indicates that the weak radiation mechanism is dominant and suggests that the coal and rock system is prone to plastic flow failure (sliding failure). $\Delta f_{m}<0$ indicates that a strong microradiation mechanism is dominant and that the coal rock system is at the stage of crack propagation.

\subsection{Time-varying response characteristics of EMR from coal rock burst}

Figure 8 shows the temporal multifractal spectrum of EMR measured in the excavation of the bolted roadway of No. 23150 mining face of Coal Mine A. In the rock burst development, $\Delta \alpha_{m}$ gradually increased from 0.2 to 0.48 . At the same time, $\Delta f_{m}$ gradually increased from -0.9 to the maximum of 0.38. After the peak, both $\Delta \alpha_{m}$ and $\Delta f_{m}$ decreased and then maintained a certain level, and $\Delta f_{m}$ fluctuated around 0. During this period, the rock burst occurred.

During roadway excavation, the variations in multifractal EMR parameters, $\Delta \alpha_{m}$ and $\Delta f_{m}$, mainly underwent three phases: the embryonic, developmental and critical phases. During the early excavation, the roadway ends were far from the stress-concentrated areas. As shown in Fig. 8, $\Delta \alpha_{m}$ was between 0.2 and 0.28 and $\Delta f_{m}$ was less than 0 ( -0.1 or so); this phase was called the embryonic phase. With the working face gradually approaching the stress-concentrated area, the difference in EMR formation mechanisms increased, coal rock gradually entered the nonlinearly accelerated deformation phase, and the rate of crack extension increased, leading to enhanced coal rock plasticity and making the coal rock system prone to shear sliding failure. At this time, $\Delta \alpha_{m}$ increased from 0.28 to 0.48 , and $\Delta f_{m}$ increased from -1.0 to the critical value 0 , that is, the coal rock system entered the developmental phase. With the excavation roadway advancing, $\Delta \alpha_{m}$ reached its peak and began to decrease, and $\Delta f_{m}$ fluctuated around the critical value 0 (from -0.5 to +0.48 ), that is, the coal rock system was in the critical phase. In this phase, the cracks developed sufficiently and the rate of their extension decreased to a certain extent; the coal rock system moved from the phase of crack-dominated damage into the phase of shear-sliding-dominated damage. The system entered into an organized critical state and formed a dissipative structure with a degree of stability; however, it was prone to overall instability under external disturbances. The rock burst event in the excavation of the bolted roadway of caving face no. 23150 occurred in this phase. After the rock burst, the energy concentrated in the surrounding rock was released and the coal rock system entered the first phase of coal rock deformation anew, and $\Delta \alpha_{m}$ and $\Delta f_{m}$ returned to their initial levels.

Figure 9 shows the temporal multifractal spectrum of EMR in the advancing face of Coal Mine B. This rock burst event occurred after the $\Delta \alpha_{m}$ reached its peak and $\Delta f_{m}$ fluctuated around 0 ; this was similar to the rock burst that occurred at the excavation roadway of Coal Mine A. Before this, both $\Delta \alpha_{m}$ and $\Delta f_{m}$ increased gradually from 0.15 to 0.27 for $\Delta \alpha_{m}$ and from -0.6 to 0 for $\Delta f_{m}$, which can be considered as the developmental phase of rock burst. It was difficult to distinguish the embryonic phase from the 


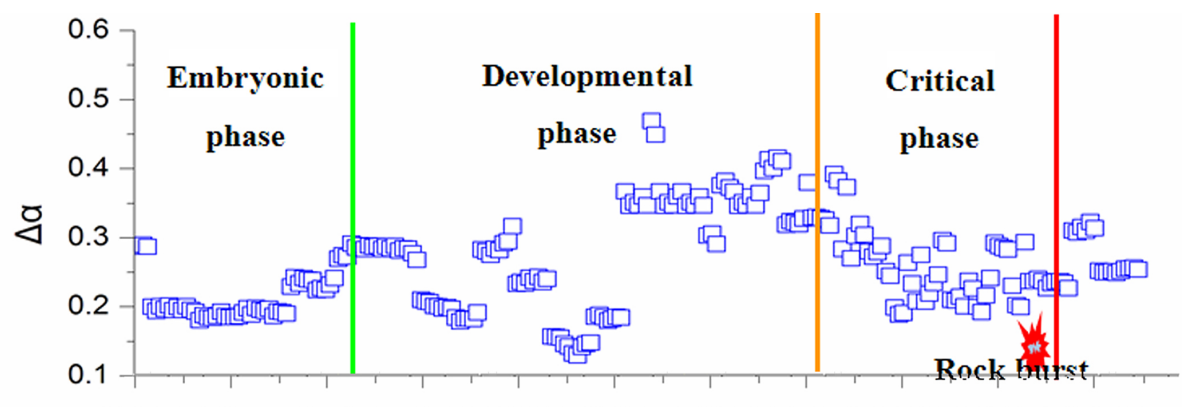

$2013-3-3 \quad 2013-3-4 \quad 2013-3-5 \quad 2013-3-6 \quad 2013-3-7 \quad 2013-3-8 \quad 2013-3-9 \quad 2013-3-10 \quad 2013-3-11 \quad 2013-3-12 \quad 2013-3-13$

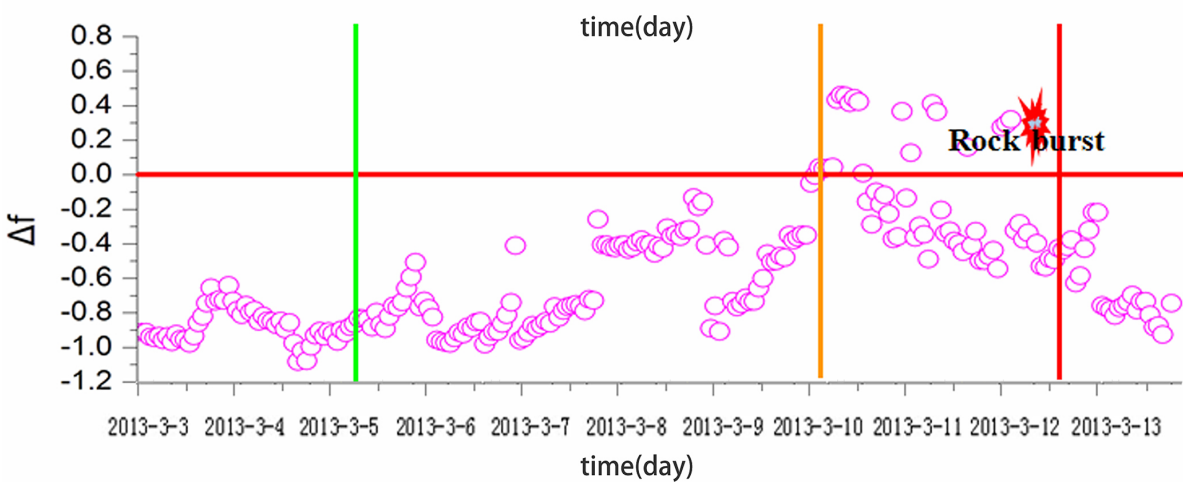

Figure 8. Temporal multifractal spectrum of EMR in roadway no. 23150 of Coal Mine A.
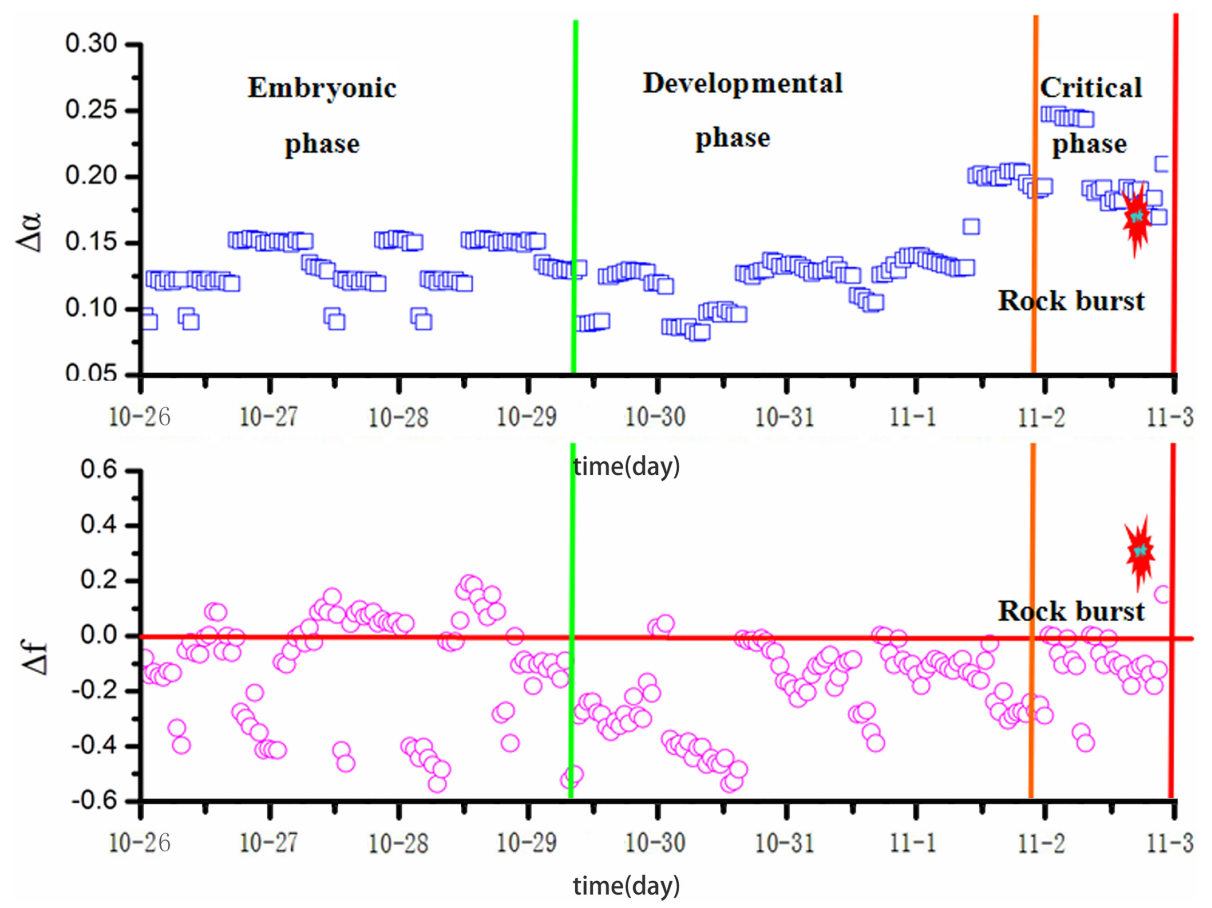

Figure 9. Temporal multifractal spectrum of EMR in the bolted roadway of the heading face of Coal Mine B.

developmental phase. In the embryonic phase, $\Delta f_{m}$ also fluctuated around 0 strongly, but $\Delta \alpha_{m}$ remained at the low level $(0.1-0.15)$. The in situ analysis indicated that Coal Mine B was already in a damaged state, and its crack development was more advanced. Thus, characteristics of both elastic compaction and microcrack nucleation stages of coal rock were not obvious. Under mining-induced stress, coal rock was prone to friction sliding failure, thus resulting in $\Delta f_{m}>0$. Only when the instantaneously released elastic strain energy of the surrounding rock is much larger than the 


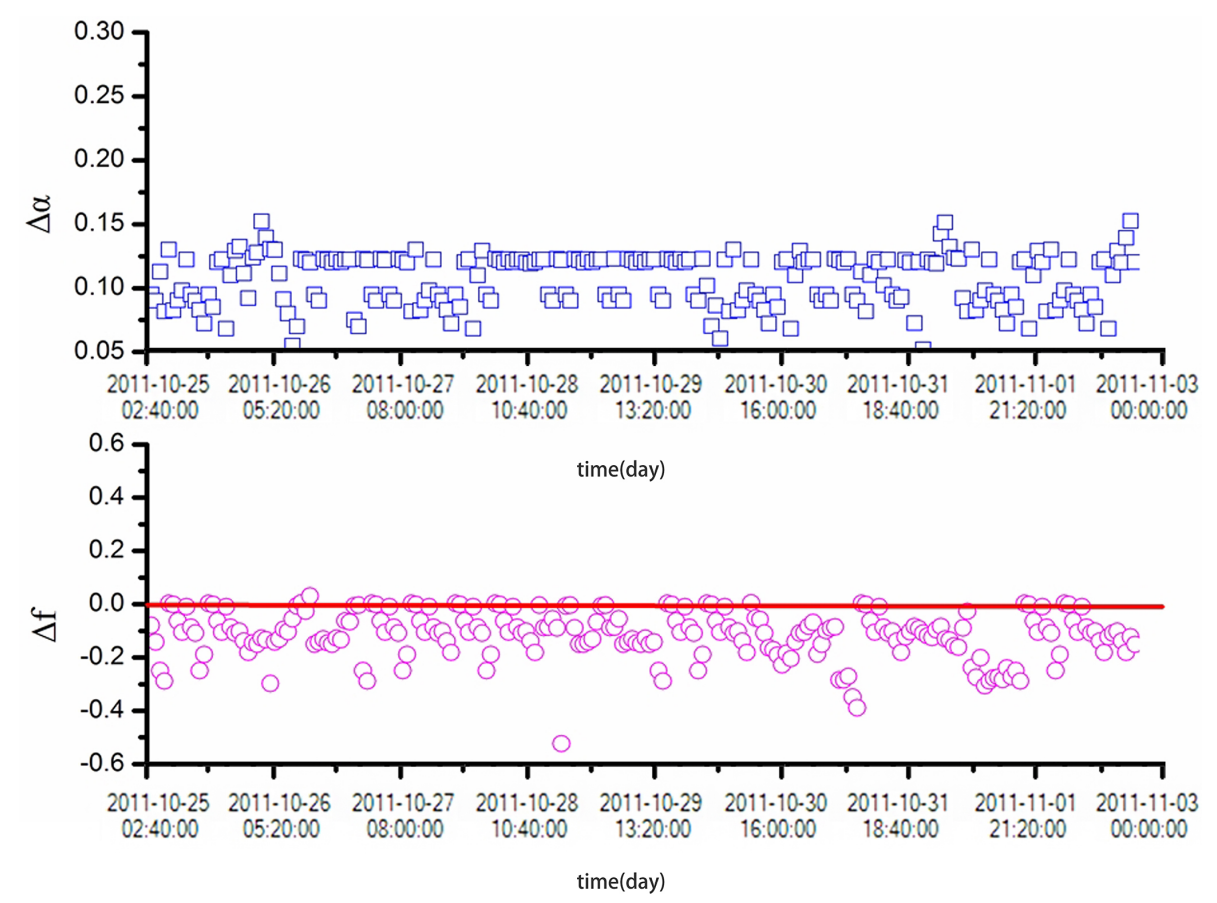

Figure 10. Temporal multifractal spectrum of EMR in non-danger zone.

friction sliding dissipated energy of coal rock, can new fracture sections be formed; at this time, $\Delta \alpha_{m}$ increases further, and $\Delta f_{m}$ is more often less than 0 (the developmental phase).

For non-rock-burst-prone areas, the variation of $\Delta \alpha_{m}$ and $\Delta f_{m}$ is different from that in the rock-burst-prone zone. $\Delta \alpha_{m}$ approximately equals 0.1 (simple fractal) and $\Delta f_{m}$ fluctuates below 0 (see in Fig. 10). Experimental phenomena indicate that coal or rock is in a stable state for non-rock-burst-prone zones, and the mechanism of electromagnetic radiation is relatively single.

From the typical examples above, it is obvious that only when the variations in $\Delta \alpha_{m}$ and $\Delta f_{m}$ are analyzed simultaneously, the phase and state of the coal rock system can be determined. Before rock burst, the system must experience the embryonic, developmental and critical phases; the temporal multifractal spectra of EMR released by coal rock in different phases are different, that is, they have their own independent characteristics.

\section{Discussions}

\subsection{Nature of time-varying multifractal spectrum of EMR from coal rock bursts}

Scholars have conducted many experiments to study the microscopic EME mechanism and found that the generation of EMR is directly or indirectly related to mechanical processes, such as the piezoelectric effect (Nitsan, 1977; Ghomshei and Templeton, 1989; Huang, 2002), the electrodynamic effect (Martelli et al., 1989), electric charge separation and relaxation (Ogawa et al., 1985; Enomoto and Hashimoto, 1990; Tzanis et al., 2000; Vallianatos et al., 2004; Rabinovitch et al., 2007), the variable motion of electric charges (Gokhberg et al., 1982; Nagahama and Teisseyre, 1998; Freund et al., 2004; Triantis et al., 2006), and the Coulomb field of the surface charges generated by the fractional sliding effect, etc. (Miura and Nakayama, 2001; Muto et al., 2006; Akito and Nobumasa, 2008). It is clear that the damage caused by several different types of failure such as crack opening, crack propagation and fractional sliding can produce EMR, but their corresponding microemission mechanisms are different. This difference is likely the principal reason for the time-varying characteristics of EMR fractals and the dynamic changes in approximate entropy.

On the basis of irreversible thermodynamics of damage evolution, Kawada et al. (2007) introduced electric dipoles and explained the critical phenomena of EM emission and the timescale invariance theoretically. Based on the law of energy conservation for the thermodynamic equilibrium system, they, in fact, established a coupling model for the EMR field and mechanical energy release during coal damage evolution, namely,

$\mathrm{d} G=-s \mathrm{~d} T-\frac{\partial G}{\partial \sigma} \mathrm{d} \sigma-\frac{\partial G}{\partial p} \mathrm{~d} p+\frac{\partial G}{\partial \alpha} \mathrm{d} \alpha$,

where $G$ is the Gibbs free energy, $s$ the entropy density (Nagahama and Teisseyre 1998), $T$ the absolute temperature, $\partial G / \partial \sigma$ the macroscopic elastic strain and $\partial G / \partial p$ the electromagnetic field $(\tilde{E})$. 
The above model is built based on the isothermal and linearly irreversible thermodynamic process, as well as on the damage statistical mechanics which do not distinguish between damage types. In fact, the dissipated energy in the material deformation and failure process is used up in the damage evolution process caused by irreversible internal variation, friction, sliding and thermal dissipation; therefore, the above model has its limitations. Although it can explain the critical phenomena of EM emission well, it is difficult to explain EM emissions dynamic multifractal characteristics.

EM emission from coal or rock is closely relevant to the stress state. The stress determines the process of coal and rock damage evolution, thus affecting EMR dynamic changes. Some scholars have already revealed the relationship between coal electromagnetic radiation and the damage evolution process. The rock damage evolution is related to the deformation and failure process of energy dissipation, and the damage evolution model could be established based on dissipative energy. Hence, EMR has an intrinsic relationship with the dissipated energy; from the point of view of thermodynamic laws and damage mechanics and based on the types of coal deformation and failure, we introduce the damage internal variable, construct a coal and rock dissipation potential function and establish the coupling model between EMR and dissipation energy, which reveals the nature of dynamic nonlinear characteristics of EMR.

According to the first law of thermodynamics, the increment of a system internal energy is equal to the difference between the heat transferred from the outside to the system and the work done by the system on its environment; it can be expressed using the following integral form

$$
\begin{aligned}
\frac{\mathrm{d}}{\mathrm{d} t} \int_{v}\left[\frac{1}{2} \rho\left(\frac{\partial u}{\partial t}\right)^{2}+e\right] \mathrm{d} v & =\int_{v} b \frac{\partial u}{\partial t} \mathrm{~d} v+\int_{s} p \frac{\partial u}{\partial t} \mathrm{~d} s \\
& +\int_{v} I \mathrm{~d} v-\int_{s} q \cdot n \mathrm{~d} s,
\end{aligned}
$$

where $v$ is the volume of the control body, $S$ its surface area, $n$ the outward normal, $e$ is the internal energy per unit volume, $\rho$ the density, $b$ the volume force, $p$ the surface force, $u$ the displacement, $I$ the intensity of heat source and $q$ the heat flux.

The differential form of the first law of thermodynamics is

$\dot{e}=\sigma \cdot \dot{\varepsilon}-I+\operatorname{div} \boldsymbol{q}$,

where $\sigma$ is the stress tensor, and $\varepsilon$ is the strain tensor.

According to the second law of thermodynamics, the entropy production is greater than or equal to 0 :

$$
\begin{aligned}
\frac{\mathrm{d} s_{t}}{\mathrm{~d} t} & =\int_{v} \dot{s} \mathrm{~d} v-\int_{v} \frac{I}{T} \mathrm{~d} v+\int_{v} \frac{1}{T} \operatorname{div} \boldsymbol{q} \mathrm{d} v \\
& +\int_{v} \frac{1}{T} \boldsymbol{g} \cdot \boldsymbol{q} \mathrm{d} v=\int_{v} \frac{\psi}{T} \mathrm{~d} v,
\end{aligned}
$$

where $\psi$ is the dissipated potential function, $s$ the total entropy, $T$ the temperature and $g$ the temperature gradient.

Putting Eq.(12) into Eq. (13), we get

$\psi=T \cdot \dot{s}-\dot{e}+\sigma \cdot \dot{\varepsilon}+\boldsymbol{g} \cdot \boldsymbol{q}$.

Introducing the Helmholtz free energy $\Phi=e-T S$ and the irreversible internal variable $\omega$, and with $\Phi=\Phi(\varepsilon e, \varepsilon p, \omega, T)$, we have

$$
\left.\begin{array}{l}
\Phi=\Phi\left(\varepsilon^{e}, \varepsilon^{p}, w, T\right) \\
\dot{\Phi}=\dot{e}-T \cdot \dot{s}-s \cdot \dot{T}
\end{array}\right\} .
$$

Because the first law of thermodynamics holds true, combining Eqs. (12) and (15) and simplifying what was obtained results in

$\psi=\sigma \cdot \dot{\varepsilon}^{p}+A \cdot \dot{w}+\boldsymbol{g} \cdot \boldsymbol{q}$,

where $A$ is the thermodynamic generalized force corresponding to the irreversible internal variable $\omega$, such as the damage expansion force, $A=-\partial \Phi / \partial \omega$.

Equation (16) is the rate of energy dissipation based on the elastic model of damage mechanics. As a measure of energy dissipation per unit time, it consists of three parts, the irreversible strain energy rate (i.e., plastic strain energy), the rate of energy corresponding to irreversible internal variable (i.e., damage extension) and the heat power; their expressions are

$$
\left.\begin{array}{rl}
\dot{U}_{s} & =\sigma \cdot \dot{\varepsilon}^{p} \\
\dot{U}_{b} & =A \cdot \dot{w} \\
\dot{U}_{r} & =\boldsymbol{g} \cdot \boldsymbol{q}
\end{array}\right\} .
$$

Accompanying the irreversible evolution process of the system, the dissipation energy can accurately reflect the state of the system. In the different phases of damage and failure of coal, the quantities and components of the energy dissipation rates in various phases are significantly different. At the beginning of the coal rock deformation and failure, the dissipated energy is mainly caused by the closing of original joint fractures and friction among lattices. With the loading increasing, coal passes into the elastic phase. At this time the energy exhausted by the plastic deformation of coal decreases, and the energy input from the outside is converted into elastic strain energy. As coal enters the plastic phase, crack expansion accelerates, accompanied by most of the energy consumption. In the post-peak failure phase, the types of energy consumption are related to coal's mechanical properties. Coals with lower intensity consume energy mainly 
through plastic slip, whereas coals with greater strength dissipate energy mainly through brittle fracture. All the phases are accompanied by different degrees of thermal dissipation.

It is clear that EMR energy is inherently related to the dissipated energy during the damage and failure of coal. Assuming that the EMR energy couples with the dissipation energy and that $\alpha, \beta$ and $\gamma$ are three coupling coefficients determined by the inherent nature of coal, then the EMR energy rate is defined as follows:

$\dot{U}_{e}=[\alpha, \beta, \gamma]\left[\begin{array}{c}\dot{U}_{s} \\ \dot{U}_{b} \\ \dot{U}_{r}\end{array}\right]=\alpha \dot{U}_{s}+\beta \dot{U}_{b}+\gamma \dot{U}_{r}$.

Equation (18) determines the EMR energy released by coal per unit time and is referred to as the electromechanically coupling model of coal EMR. Equation (18) represents the characteristic rules of the EMR signal based on energy dissipation, indicating that the EMR signal is determined by the size of the dissipated energy rate and components and is the result of combined effects of multiple influential factors. Different coal properties and system states lead to different dissipated energy rates and components in the process of deformation and fracture and thus to dynamic, nonlinear changes of EMR.

Rock burst is a dissipative structure that can exchange matter and energy with the outside world (Lu et al., 2004, 2007). In fact, it is composed of coal and rock units that have different states, or in its implicit complexity (multi-scale features and fractal structures), which leads to the spatiotemporal multifractal of EMR. The rock burst system can be described by entropy flow and entropy change. In the early development phase of rock burst, the system is nearly in an equilibrium state. At this time entropy change is slightly smaller than entropy flow (the dissipation energy is less than the input energy from the outside world), coal rock is in the elastic deformation, compaction and microcrack nucleation stage, the emitted EMR is contributed mainly by microcrack nucleation, $\Delta \alpha_{m}$ is maintained at a lower level, and $\Delta f_{m}$ is mainly controlled by the first kind of microcosmic mechanism of radiation. When the mining space advances to the stress concentration area, entropy change starts to deviate from entropy flow (the external input energy suddenly increases and is much larger than the capacity consumed by the system), crack expansion and penetration are accelerated, forming a multiple-fracture structure. At the same time, due to coal-rock-crack-induced damage, shear-stress-induced plasticity is enhanced, and the shear slip failure mode gradually increases, resulting in multifractal dimensions and dynamic changes of EMR emission: $\Delta \alpha_{m}$ gradually increases, and $\Delta f_{m}$ changes gradually from one microscopic radiation mechanism (less than 0) to another microradiation mechanism (greater than 0 ). When the system is fully in the selforganized critical state, i.e., entropy change $\approx$ entropy flow, the system has a dissipative structure with a certain degree of stability and could absorb the external input energy through self-adjusting. However, it is prone to dynamic disasters given external disturbances. At this point, cracks are sufficiently developed. EMR is controlled by two kinds of micromechanisms of radiation: $\Delta \alpha_{m}$ maintains a certain level, and $\Delta f_{m}$ fluctuates around 0 .

\subsection{Significance of the temporal multifractal spectrum of EMR from coal rock bursts}

EMR shows an obvious trend before rock burst. Rock deformation and failure mainly go through three phases: nucleation of microcracks, extension and rupture instability. EMR emanates from early onset destructive microcracks with a 1$2 \mathrm{~cm}$ width (Frid and Vozoff, 2005) before large-scale fracture. Hence, recording high-frequency EMR generated much earlier, before the collapse, could yield a significant time advantage for early warning.

In the typical examples above, EMR emitted from coal rock revealed the coal rock inner abnormality and the possibility of rock bursts in 1-2 days. However, EMR signals from these two rock burst evolution processes are obviously different. In Coal Mine A, basal EMR intensity in the advancing roadway was around $20 \mathrm{mV}$ and rock burst occurred while EMR intensity was declining. By contrast, in Coal Mine B, basal EMR intensity in the combined mining face was $150 \mathrm{mV}$ and rock burst occurred while EMR intensity was increasing. Thus, the rock burst event may occur in the process both of EMR increasing and declining. The complex nonlinear behavior of the rock burst system can not be explained by traditional theories, which have their own shortcomings in EMR monitoring and early warning.

In fact, the coal rock system is composed of coal and rock units. A local failure of the system will not necessarily cause its global failure and a burst. EMR signals released from the coal rock system originate from the comprehensive interaction among its components. EMR signals released from the processes of different failure mechanisms of single and composite samples have greater differences, as shown in Fig. 11.

EMR intensity of coal rock during deformation and rupture increases to some extent, which is not obvious in combined rock samples. The overall EMR intensity is kept around $1000 \mathrm{aJ}$ although its signal components become more abundant. For a single rock sample, the peak EMR intensity is close to $1000 \mathrm{aJ}$ and obviously only changes during deformation and rupture. This characteristic can be used to predict coal rock deformation and failure. However, EMR from combined rock samples is more complex and can not be used alone to distinguish outburst from failure. Increasing evidence has shown that it is difficult to quantitatively describe seismic electromagnetic radiation (SEMR) phenomena by "linear" models using "averaged" parameters. Because a coal rock system is highly heterogeneous (on all scales) and anisotropic, currently fractal-theoretic models are the best to describe it. 

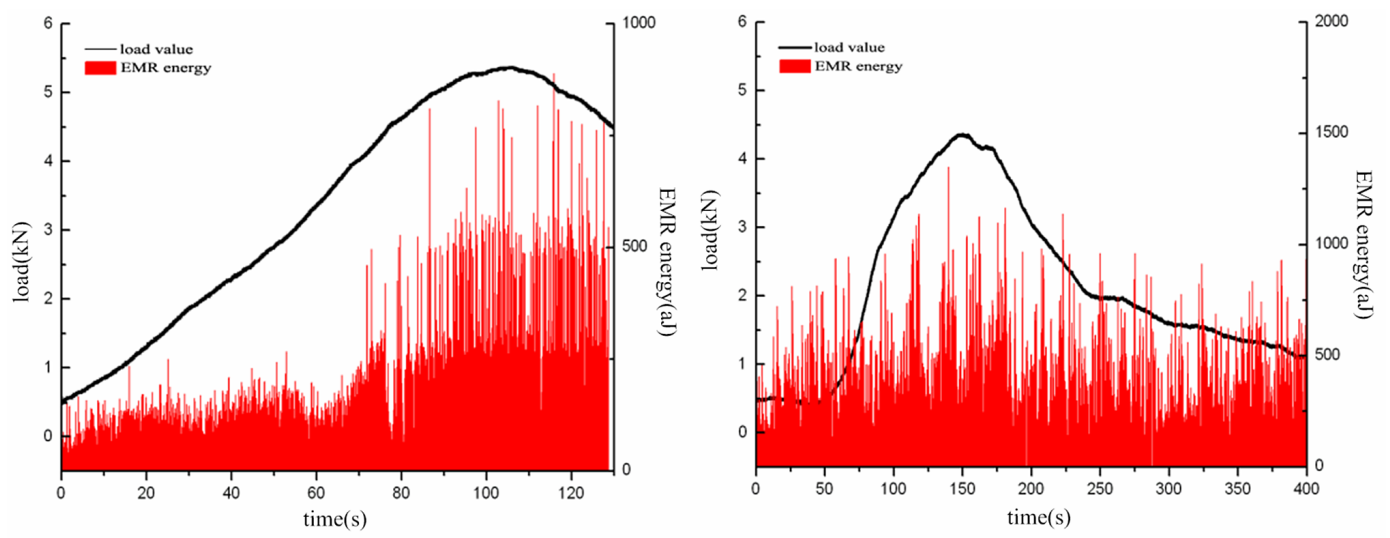

Figure 11. Changes in EMR from the coal rock unit and their combination. (a) EMR from a coal rock unit at loading rate of $0.1 \mathrm{~mm}^{\text {min }}{ }^{-1}$; (b) EMR from complex coal rock composed of four coal and rock units at loading rate of $0.1 \mathrm{~mm} \mathrm{~min}^{-1}$.

The effective solution to rock burst prediction and early warning is the use of nonlinear theories and methods to study the origination and development of rock burst and to establish corresponding prediction methods. The concept of a "fractal" was presented by Mandelbrot to assess and model irregular and complex patterns (Mandelbrot, 1982). In this paper, we proposed the time-varying multifractal spectrum distribution, which reveals the spatiotemporal dynamic character of fractal systems. We analyzed the temporal multifractal spectra of EMR from two typical coal rock bursts in deep coal mining spaces. The results showed that although EMR signals from coal rocks at different mining faces have certain differences, they may be generated by the same mechanism of rock bursts. Based on the dynamic change of multifractal parameters $\Delta \alpha_{m}$ and $\Delta f_{m}$, we can judged the stress level and state of the coal or rock more accurately. Overall, temporal response characteristics of EMR to imminent coal or rock dynamic disasters such as rock burst or roof caving, etc., in the underground mining space provide a reliable basis for issuing an early warning.

\section{Conclusions}

EMR signals generated during underground mining as the fingerprint of coal rock burst failure are closely related to microcrack nucleation $(1-2 \mathrm{~cm})$ and the expansion of coal rock and even more closely to the inception and evolution of rock bursts. Coal rock in the hazardous zone exchanges matter and energy with the external world and gradually evolves from a stable or equilibrium state to a nonequilibrium dissipative state and eventually into a self-organized, critical state. EMR generation at the mining face is mainly dominated by two types of microscopic mechanisms. The first one consists of the transient changes in stress-induced electric dipoles and electric charges due to crack-development-induced variable motion, separation and relaxation (Gokhberg et al., 1982; Nagahama and Teisseyre, 1998; Freund et al., 2004; Triantis et al., 2006). The second is the Coulomb field due to accumulation dislocation, sliding and friction of electric charges on the fracture surfaces (Miura and Nakayama, 2001; Muto et al., 2006; Akito and Nobumasa, 2008). EMR signal generation is related to the dislocation and sliding of coal joints, cracks and lattices, as well as crack development. For a coal rock system in different states, the contributions of these two mechanisms is different, resulting in nonlinear, dynamic change in EMR.

Influenced by mining stress and excavation, a coal rock system will spatiotemporally form multi-scale features and fractal structures, which in turn lead to the emission of EMR with temporal multifractal features. The temporal multifractal spectrum of EMR intrinsically carries both a spatial factor (multiple fracturing of coal rock) and a temporal factor (the dynamic, temporal evolution of fractures). Therefore, it can accurately respond to deformation, rupture and failure and be used to monitor coal rock dynamic hazards and give an early warning of these.

Temporal response characteristics of EMR show that the process of coal rock burst evolution involves inception, development and critical stages. At the inception stage, coal rock undergoes elastic compaction and microcrack nucleation. Thus, radiated EMR signals are contributed mainly by microcrack extension, meaning $\Delta \alpha_{m}$ is small and $\Delta f_{m}$ is mostly less than 0 . At the development stage, cracks rapidly expand, connect to each other and form multi-scale fissures, which further damage coal rocks and accelerate plasticity and shear sliding failure. During this period, the measured EMR signals are contributed mainly by crack extension and shear sliding, and $\Delta \alpha_{m}$ and $\Delta f_{m}$ increase gradually. At the critical stage, a coal rock system moves into the self-organized, critical state, $\Delta \alpha_{m}$ decreases slightly and $\Delta f_{m}$ fluctuates around 0 ; the system is prone to burst failure due to disturbances.

Although the dynamic multifractal spectrum of EMR is an objective response to deformation and failure of the loaded coal rock and can be used to evaluate the deformation and 
fracture process and failure of coal rocks, the fractal parameters $\Delta \alpha_{m}$ and $\Delta f_{m}$ of EMR are affected by various factors, including loading rate as well as the mechanical properties and stress levels of coal rock. Therefore, the dynamic changes in the multifractal parameters $\Delta \alpha_{m}$ and $\Delta f_{m}$ and their impacting factors need to be further investigated.

Acknowledgements. This work is supported by the Research Project of the Chinese Ministry of Education (No113031A) and a project funded by the Priority Academic Program Development of Jiangsu Higher Education Institutions and National Natural Science Foundation of China (51104156). The authors are grateful to all the coal mines mentioned in the paper for providing experimental environments.

Edited by: F. Vallianatos

Reviewed by: two anonymous referees

\section{References}

Akito, Tsutsum and Nobumasa, Shirai: Electromagnetic signals associated with stick-slip of quartz-free rocks, Tectonophysics, 450, 79-84, 2008.

Brady, B. T. and Rowell, G. A.: Laboratory investigation of the electrodynamics of rock fracture, Nature, 321, 488-492, 1986.

Contoyiannis, Y., Kapiris, P., and Eftaxias, K.: Monitoring of a preseismic phase from its electromagnetic precursors, Phys. Rev. E., 71, 1-14, 2005.

Cress, G. O., Brady, B. T., and Rowell, G. A.: Sources of electromagnetic radiation from fracture of rock samples in laboratory, Geophys. Res. Lett., 14, 331-334, 1987.

Eftaxias, K., Kapiris, P., Polygiannakis, J., Peratzakis, A., Kopanas, J., Antonopoulos, G., and Rigas, D.: Experience of short term earthquake precursors with VLF-VHF electromagnetic emissions, Nat. Hazards Earth Syst. Sci., 3, 217-228, doi:10.5194/nhess-3-217-2003, 2003.

Eftaxias, K., Panin, V. E., and Deryugin, Y. Y.: Deryugin. EvolutionEM signals before earthquakes in terms of mesomechanics and complexity, Tectonophysics, 431, 273-300, 2007.

Enomoto, Y. and Hashimoto, H.: Emission of charged particles from indentation fracture of rocks, Nature, 346, 641-643, 1990.

Freund, F., Takeuchi, A., Lau, B. W. S., Post, R., Keefner, J., Mellon, J., and Al-Manaseer, A.: Stress-induced changes in the electrical conductivity of igneous rocks and the generation of ground currents, Terr. Atmos. Ocean Sci., 15, 437-469, 2004, http://www.ocean-sci.net/15/437/2004/.

Frid, V. and Vozoff, T. K.: Electromagnetic radiation induced by mining rock failure, Int. J. Coal Geol., 64, 57-65, 2005.

Gang, Xiong, Zhang, Shuning, and Liu, Qiang: The time singularity multifractal spectrum distribution, Physica A, 391, 4727-4739, 2012.

Ghomshei, M. M. and Templeton, T .L.: Piezoelectric and a-axes fabric along a quartz vein, Phys. Earth Planet. Int., 55, 374-386, 1989.

Gokhberg, M. B., Morgounov, V. A., Yoshino, T., and Tomizawa, I.: Experimental measurement of electromagnetic emissions possibly related to earthquakes in Japan, J. Geophys. Res., 87, 7824$7828,1982$.
Gotoh, K., Hayakawa, M., Smirnova, N. A., and Hattori, A.: Fractal analysis of seismogenic ULF emissions, Phys. Chem. Earth, 29, 419-424, 2004.

Hayakawa, M., Hattori, K., Nickolaenko, A. P., and Rabinowicz, L. M.: Relation between the energy of earthquake swarm and the Hurst exponent of random variations of the geomagnetic fields, Phys. Chem. Earth, 29, 279-378, 2004.

He, Xueqiu, Nie, Baisheng, Chen, Wenxue, Wang, Enyuan, Dou, Linming, Wang, Yunhai, Liu, Mingju, and Hani, Mitrie: Research progress on electromagnetic radiation in gas-containing coal and rock fracture and its applications, Safety Science, 50, 728-735, 2012.

$\mathrm{Hu}$, Shaobin, Wang, Enyuan, Li, Zhonghui, Shen, Rongxi, and Liu, Jie: Time-Varying Multifractal Characteristics and Formation Mechanism of Loaded Coal Electromagnetic Radiation, Rock Mech. Rock Eng., doi:10.1007/s00603-013-0501-9, in press, 2013.

Huang, Q.: One possible generation mechanism of co-seismic electric signals, Proc. Jpn. Acad., 78, 173-178, 2002.

Kapiris, P. G., Eftaxias, K. A., Nomikos, K. D., Polygiannakis, J., Dologlou, E., Balasis, G. T., Bogris, N. G., Peratzakis, A. S., and Hadjicontis, V. E.: Evolving towards a critical point: A possible electromagnetic way in which the critical regime is reached as the rupture approaches, Nonlin. Processes Geophys., 10, 511524, doi:10.5194/npg-10-511-2003, 2003.

Kapiris, P. G., Balasis, G. T., Kopanas, J. A., Antonopoulos, G. N., Peratzakis, A. S., and Eftaxias, K. A.: Scaling similarities of multiple fracturing of solid materials, Nonlin. Processes Geophys., 11, 137-151, doi:10.5194/npg-11-137-2004, 2004a.

Kapiris, P. G., Eftaxias, K. A., and Chelidze, T. L.: Electromagnetic signature of prefracture criticality in heterogeneous media, Phys. Rev. Lett., 92, 1-4, 2004b.

Kawada, Y., Nagahama, H., Omori, Y., Yasuoka, Y., Ishikawa, T., Tokonami, S., and Shinogi, M.: Time-scale invariant changes in atmospheric radon concentration and crustal strain prior to a large earthquake, Nonlin. Processes Geophys., 14, 123-130, doi:10.5194/npg-14-123-2007, 2007.

Lu, C., Dou, L., and Xie, Y.: Experimental simulation of drilling impact of coal sample in three-axle enclosed pressure, J. China Coal Soc., 29, 660-662, 2004.

$\mathrm{Lu}, \mathrm{C}$., Dou, L., and $\mathrm{Wu}, \mathrm{X}$.: Experimental research on rules of rockburst tendency evolution and acoustic-electromagnetic effects of compound coal-rock samples, Chin. J. Rock Mech. Eng., 26, 2549-2555, 2007.

Mandelbrot, B. B.: The Fractal Geometry of Nature, W. H. Freemann and Company, San Francisco, 1982.

Martelli, G., Smith, P. N., and Woodward, A. J.: Light, radio frequency emission and ionization effects associated with rock fracture, Geophys. J. Int., 98, 397-401, 1989.

Masugi, M. and Takuma, T.: Multi-fractal analysis of IP-network traffic for assessing time variations in scaling properties, Physica D, 225, 119-126, 2007.

Minadakis, G., Stylianos, M., Potirakis, C. N., and Eftaxias, K.: Linking electromagnetic precursors with earthquake dynamics: An approach based on nonextensive fragment and self-affine asperity models, Physica A, 391, 2232-2244, 2012. 
Miura, T. and Nakayama, K.: Two-dimensional spatial distribution of electric-discharge plasma around a frictional interface between dielectric surfaces, Appl. Phys. Lett., 78, 2979-2981, 2001.

Morgounov, V. A. and Malzev, S. A.: A multiple fracture model of pre-seismic electromagnetic phenomena, Tectonophysics, 431, 61-72, 2007.

Muto, J., Nagahama, H., Miura, T., and Arakawa, I.: Frictional discharge plasma from natural semiconductor/insulator junctions: origin of seismo-electromagnetic radiation, Phys. Chem. Earth, 31, 346-351, 2006.

Muto, J., Nagahama, H., Miura, T., and Arakawa, I.: Fricitional discharge an fault asperities: Origion of fractal seismoelectromagnetic radiation, Tectonophysics, 431, 113-122, 2007.

Nagahama, H. and Teisseyre, R.: Thermodynamics of line defects and transient electric current: electromagnetic field generation in earthquake preparation zone, Acta Geophys. Pol., 46, 35-54, 1998.

Nanjo, K. and Nagahama, H.: Fractal properties of spatial distributions of aftershocks and active faults, Chaos Solit. Fract., 19, 387-397, 2004a.

Nanjo, K. and Nagahama, H.: Discussions on fractals, aftershocks, and active faults: Diffusion and seismo-electromagnetism, Arab. J. Sci. Eng., 29, 147-167, 2004b.

Nitsan, V.: Electromagnetic emission accompanying fracture of quartzbearing rocks, Geophys. Res. Lett., 4, 333-335, 1977.

Ogawa, T., Oike, K., and Miura, T.: Electromagnetic radiations from rocks, J. Geophys. Res., 90, 6245-6249, 1985.

Potirakis, S. M., Minadakis, G., and Eftaxias, K.: Analysis of electromagnetic pre-seismic emissions using Fisher information and Tsallis entropy, Physica A, 391, 300-306, 2012.

Rabinovitch, A., Frid, V., and Bahat, D.: GutenbergRichter-type relation for laboratory fracture-induced electromagnetic radiation, Phys. Rev. E., 65, 011401, doi:10.1103/PhysRevE.65.011401, 2001.

Rabinovitch, A., Frid, V., and Bahat, D.: Surface oscillations - A possible source of fracture induced electromagnetic radiation, Tectonophysics, 431, 15-21, 2007.

Song, Dazhao, Wang, Enyuan, Li, Nan, Jin, Mingyue, and Xue, Shipeng: Rock burst prevention based on dissipative structure theory, Int. J. Ming Sci. Technol., 22, 159-163, 2012.

Triantis, D., Stavrakas, I., Anastasiadis, C., Kyriazopoulos, A., and Vallianatos, F.: An analysis of pressure stimulated currents (PSC), in marble samples under mechanics stress, Phys. Chem. Earth, 31, 234-239, 2006.

Tzanis, A. and Vallianatos, F.: A critical review of electric earthquake precursors, Anna. Geof., 44, 429-460, 2001.

Tzanis, A. and Vallianatos, F.: A physical model of electrical earthquake precursors due to crack propagation and the motion of charged edge dislocations, in: Seismo Electromagnetics: Lithosphere-Atmosphere-Ionosphere Coupling, edited by: Hayakawa, M. and Molchanov, O. A., TERRAPUB, Tokyo, 117130, 2002.
Tzanis, A., Vallianatos, F., and Gruszow, S.: Identification and discriminationof transient electrical earthquake precursors: Fact,fiction and some possibilities, Phys. Earth Planet. Int., 121, 223-248, 2000.

Uritsky, V., Smirnova, N., Troyan, V., and Vallianatos, F.: Critical dynamics of fractal fault systems and its role in the generation of pre-seismic electromagnetic emissions, Phy. Chem. Earth, 29, 473-480, 2004.

Vallianatos, F. and Nomikos, K.: Seismogenic Radioemissions as Earthquake Precursors in Greece, Phys. Chem. Earth, 23, 953957, 1998.

Vallianatos, F. and Tzanis, A.: Electric current generation associated with the deformation rate of a solid: preseismic and coseismic signals, Phys. Chem. Earth, 23, 933-938, 1998.

Vallianatos, F. and Tzanis, A.: On the nature, scaling and spectral properties of pre-seismic ULF signals, Nat. Hazards Earth Syst. Sci., 3, 237-242, doi:10.5194/nhess-3-237-2003, 2003.

Vallianatos, F., Triantis, D., Tzanis, A., Anastasiadis, C., and Stavrakas, I.: Electric earthquake precursors: from laboratory results to field observations, Phys. Chem. Earth, 29, 339-351, 2004.

Wang, E.: The effect of EME and AE during the fracture of coal containing gas and its applications, Ph.D. thesis, China University of Ming and Technology, Xu Zhou, 1997.

Wang, E., He, X., Liu, X., and Zhao, E.: Technology and application of coal and rock electromagnetic radiation. Science press, BeiJing, China, 2009.

Wang, E., He, X., and Liu, X.: A non-contact mine pressure evaluation method by electromagnetic radiation, J. Appl. Geophys., 75, 338-344, 2011.

Wang, E., He, X., and Liu, X.: Comprehensive monitoring technique based on electromagnetic radiation and its applications to mine pressure, Safety Science, 50, 885-893, 2012.

Wang, Y. and Zhu, Y. S.: A short-time multifractal approach for arrhythmia detection based on fuzzy neural network, IEEE T. Biomed. Eng., 48, 989-995, 2001.

Warwick, J. W., Stoker, C., and Meyer, T. R.: Radio emission associated with rock fracture: possible application to the great Chilean earthquake of May 22, 1960, J. Geophys. Res., 87, 2851-2859, 1982.

Wei, Jian-ping, He, Xue-qiu, Wang, En-yuan, and Liu, Zhen-Tang: Electromagnetic emission multi-fractal characteristic of coal and gas outburst, J. Liaoning Tech. Univers., 24, 1-4, 2005.

Yao, Jingming, Yan, Yongye, Shui, Guohong, Yao, Junwei, and Li, Shengzhuo: Study of fractal characteristics of electromagnetic emission during coal and rock mass fracture, Chin. J. Rock Mech. Eng., 29, 4103-4107, 2010. 